Review Article

\title{
Novel Nano-Based Drug Delivery Systems Targeting Hepatic Stellate Cells in the Fibrotic Liver
}

\author{
Devaraj Ezhilararasan $\mathbb{D}^{1},{ }^{1}$ Thangavelu Lakshmi $\mathbb{D},{ }^{1}$ and Biond Raut $\mathbb{D}^{2}$ \\ ${ }^{1}$ Department of Pharmacology, The Blue Lab, Molecular Medicine and Toxicology Division, Saveetha Dental College, \\ Saveetha Institute of Medical and Technical Sciences (SIMATS), Chennai, Tamil Nadu 600077, India \\ ${ }^{2}$ Department of Pharmacology, Kathmandu Medical College Bhaktapur Duwakot, Kathmandu University, Dhulikhel, Kavre, Nepal
}

Correspondence should be addressed to Thangavelu Lakshmi; adiasdc@saveetha.com and Biond Raut; rautbiond@gmail.com

Received 26 August 2021; Revised 25 September 2021; Accepted 30 September 2021; Published 22 October 2021

Academic Editor: Venkat Kumar S

Copyright (C) 2021 Devaraj Ezhilararasan et al. This is an open access article distributed under the Creative Commons Attribution License, which permits unrestricted use, distribution, and reproduction in any medium, provided the original work is properly cited.

\begin{abstract}
Hepatic stellate cells (HSCs) exist in the liver's perisinusoidal space, are phenotypically activated, and acquire myofibroblast-like phenotype. This phenotypic transformation is accountable for the accumulation and production of various extracellular matrix (ECM) proteins, involving different fibril-forming collagens in the perisinusoidal space, producing altered hepatic function and portal hypertension and increased vascular resistance, fibrosis, cirrhosis, and hepatocellular carcinoma. The activated HSCs/myofibroblasts are principal collagen-producing cells in the damaged liver. Therefore, fibrosis treatments are often targeting HSCs. HSCs store most of the total body's retinol in their cytoplasm, and hence, antifibrotic nanomedicines are often targeted with vitamin A decoration. Vitamin A-decorated nanomedicines with siRNAs for transforming growth factor-beta, collagen, and connective tissue growth factors target to inhibit fibrogenesis and the ECM-associated gene expressions, leading to fibrosis regression. Similarly, a variety of miRNAs play pro- and antifibrotic function. In the fibrotic liver, the profibrotic miRNAs are targeted with their respective antagomir and the antifibrotic miRNAs are targeted with their respective agomirs along with HSC-specific nanodecoration. These miRNA treatments reduce fibrogenesis by downregulation of ECM-related gene expressions. However, liver fibrosis is caused by the upregulation of a different type of profibrotic signaling pathways associated with ECM accumulation in the fibrotic liver. Therefore, specific gene silencing by siRNAs or targeting particularly miRNA may also not effectively reduce fibrosis to a greater extent. However, nanodecoration of a drug is useful to deliver drugs into activated HSCs in the injured liver. Therefore, the aim of this review is to focus on targeted drug delivery towards activated HSCs in the persistently damaged liver.
\end{abstract}

\section{Introduction}

Chronic liver diseases are the main cause of universal health problem. Approximately, 2 million annual deaths are attributed to cirrhosis and its associated complications, hepatocellular carcinoma (HCC) and viral hepatitis [1]. Hepatic fibrosis is the common sequel of chronic liver diseases. If fibrosis is not properly treated, then it progresses into cirrhosis and its related complications like portal hypertension and HCC. Cirrhosis is considered as the most advanced stage of hepatic fibrosis. Cirrhosis and HCC rank $11^{\text {th }}$ and $16^{\text {th }}$, respectively, in causing global deaths. Together, HCC and cirrhosis account for 3.5\% of mortalities worldwide [1, 2]. Liver fibrosis is caused by chronic injury due to chronic ethanol intake, hepatitis infections, and metabolic disorders [3]. Fibrosis of the liver is classically distinguished by the increased synthesis and a net accumulation of a variety of extracellular matrix, comprising collagens I, III, and V, elastin, tenascin, and fibronectin in the perisinusoidal space of the liver [4]. Thus, increased ECM synthesis and scar formation are considered as the initial event of liver fibrosis mediated by the hepatic stellate cell (HSC), which is the principal collagen-producing cell in the chronically injured liver [5]. Over the last two to three decades, our understanding of the pathophysiology of fibrosis is instrumental; several studies have identified potential antifibrotic 
targets either to halt progression or reverse fibrosis [6]. Chronic liver diseases are often reversible after the withdrawal of underlying etiology, and their treatment mainly focused on etiology-specific. However, presently, no accepted antifibrotic treatments are available for human use [7]. The hepatic stellate cells (HSCs) are strategically placed in the perisinusoidal area of the liver in such a way that they communicate with hepatocytes, immune cells, and sinusoidal endothelial cells in the liver. Hence, HSCs are one of the prime targets for treating fibrotic liver [8]. However, the development of natural and synthetic antifibrotic drugs targeting HSCs in the fibrotic liver has faced challenges such as poor solubility, lack of HSC-specific targeted delivery, and less effectiveness [9]. Thus, studies have principally targeted HSCs with several nanoformulations to control or regress the ongoing liver fibrosis. This review is primarily focusing on novel nano-based drug delivery systems targeting HSCs in the fibrosed liver.

\section{Hepatic Stellate Cells}

Hepatic stellate cells exist in the perisinusoidal space or Disse space in the liver. Upon chronic liver injury, HSCs are phenotypically transformed and activated into myofibroblasts. This process is termed as HSC activation [10]. The activated myofibroblasts/HSCs are liable for the expression and secretion of numerous chemokines and profibrotic cytokines [11]. Thus, activated HSCs retain the pathophysiological status of the damaged liver by expressing plateletderived growth factors (PDGF), transforming expressing growth factor- $\beta$ (TGF- $\beta$ ), retinol binding protein (RBP) receptor, Krüppel-like factor 4 , endothelin-1, and $\alpha$-smooth muscle actin, modulating a variety of tissue inhibitors of metalloproteinases (TIMPs) and matrix metalloproteinase (MMPs). This process is known as perpetuation. In a normal liver, HSCs are quiescence and are liable for the preservation of normal ECM homeostasis. However, the phenotypically activated myofibroblasts or activated HSCs are liable for the accumulation and synthesis of an enormous amount of ECM to substantiate the ongoing injury of the liver [12]. Anatomically, the excessive ECM deposition in the perisinusoidal space of the chronically injured liver hinders the solute transport between hepatocytes and liver sinusoidal endothelial cells (LSECs), heading to functional alteration of the liver and an increase in intrahepatic resistancemediated portal hypertension [13]. Functionally, activated HSCs interact with several cell types in the perisinusoidal milieu by releasing PDGF, TGF- $\beta$, TIMPs, MMPs, endothelin-1, and others [14]. These signaling molecules from activated HSCs, act on themselves via an autocrine manner also activate other cells such as quiescent HSCs, hepatocytes, and LSECs via a paracrine manner. For instance, activated HSCs and LSECs communicate each other with ET-1, leading to portal hypertension [15]. Activated HSCs induce the proliferation and activation of quiescent HSCs by expressing PDGF and TGF- $\beta$, which increases the burden of ECM synthesis. The role of HSCs in the normal and injured liver is presented in Figure 1.

\section{Existing Strategies Targeting Activated HSCs in the Fibrotic Liver}

Since HSCs perform a pivotal role in fibrogenesis, several studies have targeted HSCs to regress fibrogenesis [16]. Accumulating experimental evidence suggests that the liver fibrosis process can be controlled by decreasing HSC activation and perpetuation. Previous studies have targeted HSCs for therapeutic purpose in the chronically injured liver by (i) decreasing the HSC proliferation via PDGF signaling, (ii) decreasing fibrogenesis through inhibition of TGF- $\beta$ signaling, (iii) targeting HSCs to promote the ECM degradation by modulating MMPs and TIMPs, (iv) inducing the clearance of activated HSCs by apoptosis, (v) inducing senescence of activated HSCs, (vi) inhibiting the proliferation of activated HSCs by halting their cell cycle, (vii) suppressing the parenchymal and qHSC activation by removal of underlying etiologies, (viii) neutralizing the oxidative stress environment of the injured liver by using antioxidants, (ix) silencing the activated HSC genes responsible for ECM synthesis, (x) targeting the profibrotic miRNAs in activated HSCs via antagomirs, (xi) decreasing epigenetic alteration in activated HSCs, and (xii) decreasing the extracellular vesicles released from activated HSCs [17-23]. The above strategies were employed by previous experimental studies by using natural and synthetic drug candidates to regulate the activated HSCs in the chronically damaged liver. However, these drugs have several disadvantages like poor bioavailability and solubility, less targeted, and off-target effects on drug delivery. The liver contains heterogenous cell population, predominantly HSCs, hepatocytes, LSECs, Kupffer cells, portal fibroblasts, and other non-resident immune cells. However, HSCs activation often leads to the overexpression of several cell surface receptors such as collagen type 4 , TGF- $\beta$, connective tissue growth factor receptors, PDGFR $\beta$, RBP receptor, synaptophysin, cluster of differentiation 44 (CD44), insulin-like growth factor-II receptor (IGF2R), and low-density lipoprotein receptor [24]. In previous studies, these receptors have been primary targeted to direct nano-based drugs towards activated HSCs. Therefore, targeting phenotypically activated HSCs in the damaged liver by nano-based drug preparation could be one of the possible strategies to increase the targeted drug delivery to HSCs. Thus, HSC-specific nanodrug construction may direct the drug towards activated HSCs in the fibrosed liver.

\section{Nanotargeting of Activated HSC Surface Proteins and Signaling}

Conventionally, inorganic nanoparticles like silver, gold, titanium dioxide, silicon dioxide, and cerium oxide are used without targeting activated HSCs in the fibrotic liver [25, 26]. Later, nanocarriers like liposomes and hyaluronic acid micelles are used to carry antifibrotic drugs and target HSCs in the injured liver $[27,28]$. These nanocarriers targetedly deliver nanoparticles into activated HSCs in the fibrotic liver. During chronic liver injury CD44 and phenotypic receptors are increasingly expressed after HSC activation. Therefore, CD44 ligands are previously targeted along with 


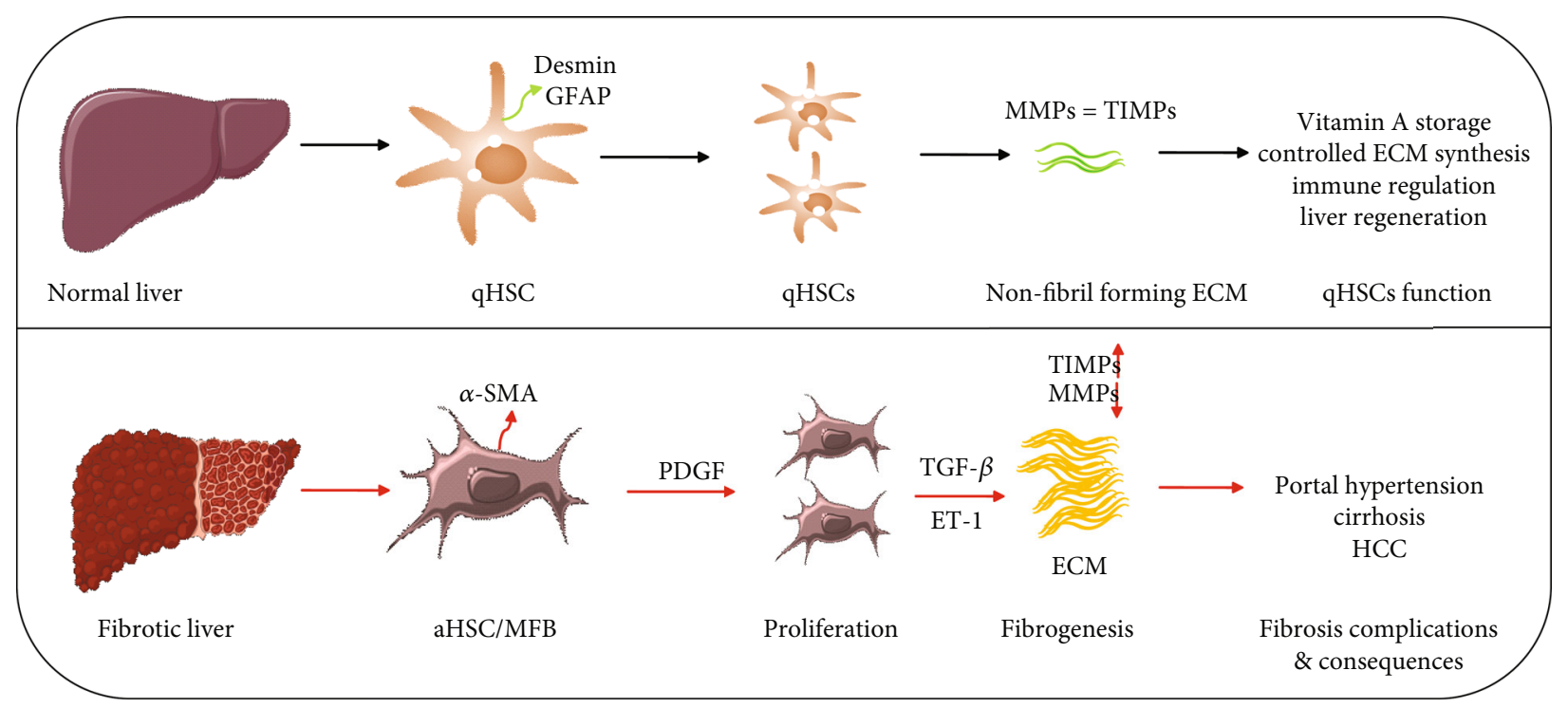

Figure 1: Hepatic stellate cells (HSCs) in the normal and fibrotic liver. In normal liver, quiescent HSC (qHSC) stores vitamin A and it is also responsible for the secretion of non-fibril-forming collagens, which gives functional support to the liver. In the fibrotic liver, qHSCs are phenotypically activated (activated HSCs (aHSCs)) and acquire myofibroblast (MFB) phenotype. The aHSCs are responsible for the enhanced secretion of extracellular matrix (ECM) via modulation of matrix metalloproteinases (MMPs) and tissue inhibitor of metalloproteinases (TIMPs). GFAP: glial fibrillary acidic protein; $\alpha$-SMA: alpha-smooth muscle actin; PDGF: platelet-derived growth factor; TGF- $\beta$ : transforming growth factor-beta; ET-1: endothelin 1; HCC: hepatocellular carcinoma.

nanoparticles to precisely deliver numerous synthetic and natural antifibrotic agents [29]. For instance, $\alpha \mathrm{v} \beta 3$ integrin is greatly expressed in activated HSCs and degradation of $\alpha \mathrm{v} \beta 3$ ligands was found in activated HSCs during the resolution of liver fibrosis. Therefore, a previous study targeted $\alpha \mathrm{v} \beta 3$ on activated HSCs [30]. Hedgehog (Hh) signaling p,lays a pivotal role in the pathophysiology of liver fibrosis [24]. Hedgehog signaling components and receptors Ihh, smoothened (Smo), patched (Ptc), and glioma-associated oncogene (Gli) 2 and Gli3 were highly expressed in HSCs [31]. Sterically steady liposomes that comprise the cyclic peptides (cRGDyK) with an excessive attraction to $\alpha \mathrm{v} \beta 3$ were developed to attain precise delivery to activated HSCs. CRGDyK-guided liposomes were specifically adopted into activated HSCs in vivo and in vitro. However, cRGDyK-guided liposomes uptake was minimal in quiescent HSCs, Kupffer cells, hepatocytes, biliary cells, or sinusoidal endothelial cells. Vismodegib (GDC), a Hh inhibitor loaded with cRGDyK-directed liposomes, prevented $\mathrm{Hh}$ signaling only in activated HSCs. In vivo, GDC-loaded cRGDyK-guided liposome treatment attenduated thioacetamide induced liver fibrosis in mice or attenuated bile duct ligation (BDL) [32]. Peroxisome proliferator-activated receptor $\gamma$ (PPAR- $\gamma$ ) overexpression prevents HSC activation in the damaged liver [33]. Rosiglitazone (RGZ), a PPAR- $\gamma$ agonist, was shown to alleviate liver fibrosis via HSC activation [34]. Therefore, methoxy-polyethylene-glycol-b-poly(carbonateco-lactide) [mPEG-b-p(CB-co-LA)] was produced and encapsulated GDC and RSZ with the encapsulation efficiency being $5.0 \%$ and $2.0 \% w / w$ for GDC and RSZ, respectively, to target against BDL-caused liver fibrosis in rats. Systemic administration of drug-encapsulated nanoparticles (120-130 nm-sized) protected liver injury in BDL-induced liver fibrosis by decreasing inflammatory cytokines and controlling the activation of
HSCs. GDC- and RSZ-loaded nanoparticles reduced $\mathrm{Hh}$ ligand molecule (Shh, Smo, Ptch-1, and Gli 1) expression and fibrosis marker expressions ( $\alpha$-SMA, fibronectin-1, and TGF- $\beta 1$ ) [35]. These studies suggest that nanoparticles with Hh inhibitor and PPAR- $\gamma$ agonist could target activated HSCs and reduce the fibrogenic potential.

Interestingly, activated HSC integrin was targeted using plant-derived active compound quercetin and along with gadolinium. RGD-hepatitis B core $(\mathrm{HBc}) / q u e r c e t i n$ and RGD-HBc/quercetin-gadolinium nanocages $(29.6 \pm 3.1 \mathrm{~nm})$ were prepared with the surface-presented RGD-directing ligand. These nanocages display high binding attraction with integrin $\alpha \mathrm{v} \beta 3$ and thereby achieved targeted delivery towards activated HSCs. These nanocages effectively prevented the HSC proliferation and their activation in vitro. This study shows that multifunctional $\alpha \mathrm{v} \beta 3$-directed NPs could deliver quercetin to the activated HSCs [36]. Germacrone (GMO), a major bioactive component of Curcuma zedoaria, and miR-29b, an antifibrotic miRNA encapulated into nanoparticles based on poly(ethylene glycol)-block-poly(lactide-co-glycolide) (PEG-PLGA), were developed with cyclical RGD peptides $(231.2 \mathrm{~nm}$ size) for an active ligand to integrin $\alpha v \beta 3$ binding. This nanoparticle system efficiently internalized into HSCs via $\alpha \mathrm{v} \beta 3$-mediated endocytosis. GMO and miR-29b inhibit the proliferation of activated HSCs and collagen 1 expression, and in vivo, this nanoparticle system ameliorated $\mathrm{CCl}_{4}$ - induced liver fibrosis in mice [37].

\section{Vitamin A- (VA-) Coupled Nanoparticles Targeting activated HSCs}

HSCs store $80 \%$ of the body's total retinol existing as retinyl esters inside their lipid droplets. Vitamin A storage is a characteristic feature of HSCs [38], and therefore, this property 
has been applied as a mechanism for targeting HSCs. Most of the natural antifibrotic compounds have poor solubility and bioavailability [9]. Hence, to achieve targeted drug delivery to HSCs, VA decoration may be a useful strategy to direct the antifibrotic compounds towards activated HSCs in the damaged liver [3, 39]. In a study, zein/phospholipid composite NPs coupled with VA $(192 \mathrm{~nm})$ were formed as a delivery platform for gallic acid (GA-CACNP/VA), a polyphenolic compound, reportedly having antifibrotic effects. These NPs showed high (60\%) entrapment efficiency (EE\%), selectively distributed in the liver and highly internalized into HSCs. GA-CACNP/VA evidently inhibited HSC activation, induced activated HSC apoptosis, and repressed collagen deposition [40]. This study implies that VA decoration can lead to HSC-specific efficient drug delivery in the injured liver. In a study, retinol was conjugated with polyethyleneimine, which further combined with an antisense oligonucleotide to form retinol-conjugated polyethyleneimine (RCP) nanoparticles. Retinol conjugation caused specific recruitment of retinolconnecting protein in the corona that effectively directed the nucleotide-laden nanoparticles into HSCs in the fibrotic liver. The oligonucleotide-laden RCP nanoparticles suppressed collagen 1 expression and attenuated $\mathrm{CCl}_{4}$ - and BDL-caused liver fibrosis in mice [41].

Imatinib, a second-generation tyrosine kinase inhibitor (TKI), has been significantly employed for the treatment of HCC, gastrointestinal stromal tumors, and chronic myelogenous leukemia [42]. Imatinib was shown to inhibit TGF- $\beta$ signaling and profibrotic PDGF pathways [43]. Therefore, VA-combined imatinib-rich liposomes (spherical and 71.9 $\pm 2.6 \mathrm{~nm}$-sized) have been targeted against HSCs in the fibrotic liver in rats. The VA-coupled imatinib liposomes are acumulated in the fibrotic liver when compared to the normal liver in rats. A single intraperitoneal injection of this liposome had 13.5 folds higher hepatic accumulation. Colocalization of VA-coupled liposome-rich Nile Red with immune-fluorescently labeled PDGFR $\beta$ in frozen liver tissue sections confirmed HSC directing [44]. The acumulation of different ECM in the liver perisinusoidal space is the chief pathological feature of liver fibrosis [8]. Thus, acceleration of matrix degradation is considered one of the therapeutic strategies for fibrosis regression. In a study, collagenase I, a collagen 1-degrading enzyme, and retinol-codecorated polymeric micelle that possesses nano-drill-like and HSC target function based on poly-(lactic-co-glycolic)-b-poly(ethylene glycol)-maleimide (PLGA-PEG-Mal) (CRM) was prepared. The spherical CRM $(87.6 \pm 8.3 \mathrm{~nm})$ had a nano-drill-like function and effectively degraded pericellular collagen I. CRM loaded with nilotinib, a second-generation TKI, showed antifibrotic activity against $\mathrm{CCl}_{4}$-induced liver fibrosis [45].

\section{Nanotargeting of activated HSCs for Portal Hypertension}

Increased intrahepatic resistance and portal hypertension are major complications of advanced fibrosis and cirrhosis. HSC activation and subsequent ET-1 release from myofibroblasts and LSECs cause portal hypertension [15]. The influence of angiotensin II receptor antagonist, i.e., valsartan, on portal hypertensiand hepatic fibrosis has been studied in hepatic cirrhotic patients [46, 47]. Valsartan reduced portal hypertension in hepatic cirrhosis patients [48]. However, valsartan exhibits poor water-soluble $(0.18 \mathrm{mg} / \mathrm{ml})$ property and it has $\sim 23 \%$ of oral bioavailability [49]. Therefore, liposome-based nanocarriers for valsartan were developed to improve its permeability. These valsartan-loaded liposomes (VLL) (spherical and 169.9 $\pm 2.4 \mathrm{~nm}$-sized) are coupled with VA for HSC specific targeted delivery. The orally administered VLL preferentially accumulated in the fibrotic liver for six days [50]. Further, reduced PPAR- $\gamma$ nuclear receptor expression was reported during HSC activation, while its upregulation was responsible for the regression of fibrosis and downregulation of fibrosis-associated gene expressions and fibrosis resolution [33]. VLL exhibits poteintal antifibrotic effects with PPAR- $\gamma$ agonistic activity and prolonged delivery [50]. Nitric oxide is a potent vasodilator, and it was shown to inhibit HSC activation and regulate portal hypertension [15]. Therefore, nitric oxide (NO) donor molecule-based drug delivery systems have been developed. For instance, silica and gold nanoparticlearbitrated drug delivery systems containing NO donors have been tried against liver fibrosis. NO released from silica and gold nanoconjugates inhibited HSC proliferation, activation, and their vascular tube formation ability [51]. Similarly, polymeric nanoparticles incorporating nitric oxide donor molecules (S-nitrosoglutathione), conjugated with VA $(25-35 \mathrm{~nm}$-sized), were studied in vitro and in vivo. The VA decoration led to the polymeric nanoparticles specifically target rat primary HSCs and LX-2 cells. This nanoparticle system significantly inhibited fibrogenic marker gene expressions such as collagen and $\alpha$-SMA without inducing cytotoxicity in primary HSCs isolated from rats and in human HSC cell line (LX-2). Further, NO-delivering nanoparticles have reduced the ET-1 and portal pressure (approximately 20\%) in BDL rats [52]. These studies indicate that gold, silver, and polymeric nanoparticle system may serve as a novel delivery platform to deliver anti-hypertensive drugs and vasodilators like NO into activated HSCs responsible for portal hypertension during chronic liver diseases.

\section{RNA Interference-Based Targeted Drug Delivery in liver fibrosis}

RNA interference is one of the valuable approaches to regress fibrosis of the liver by silenceing specific gene expression in activated HSCs [53]. The activated HSCs are liable for the accumulation of a variety of ECM proteins in the perisinusoidal space of chronically injured liver, which causes liver fibrosis. Therefore, ECM-specific gene silencing may reduce the ECM accumulation in the liver, decreasing fibrosis and associated complications [54]. siRNA-based nanopreparation offers promissing and targeted drug delivery. For instance, siRNA-mediated interference of poly $(\mathrm{rC})$ binding protein 2 (PCBP2) gene inhibits the expression of $\alpha$-complex protein-2 ( $\alpha \mathrm{CP} 2)$ in HSCs, which is accountable for stabilization of collagen $\alpha 1$ (I) mRNA. The decay rate of the collagen $\alpha 1(\mathrm{I})$ mRNA was shown to increase 


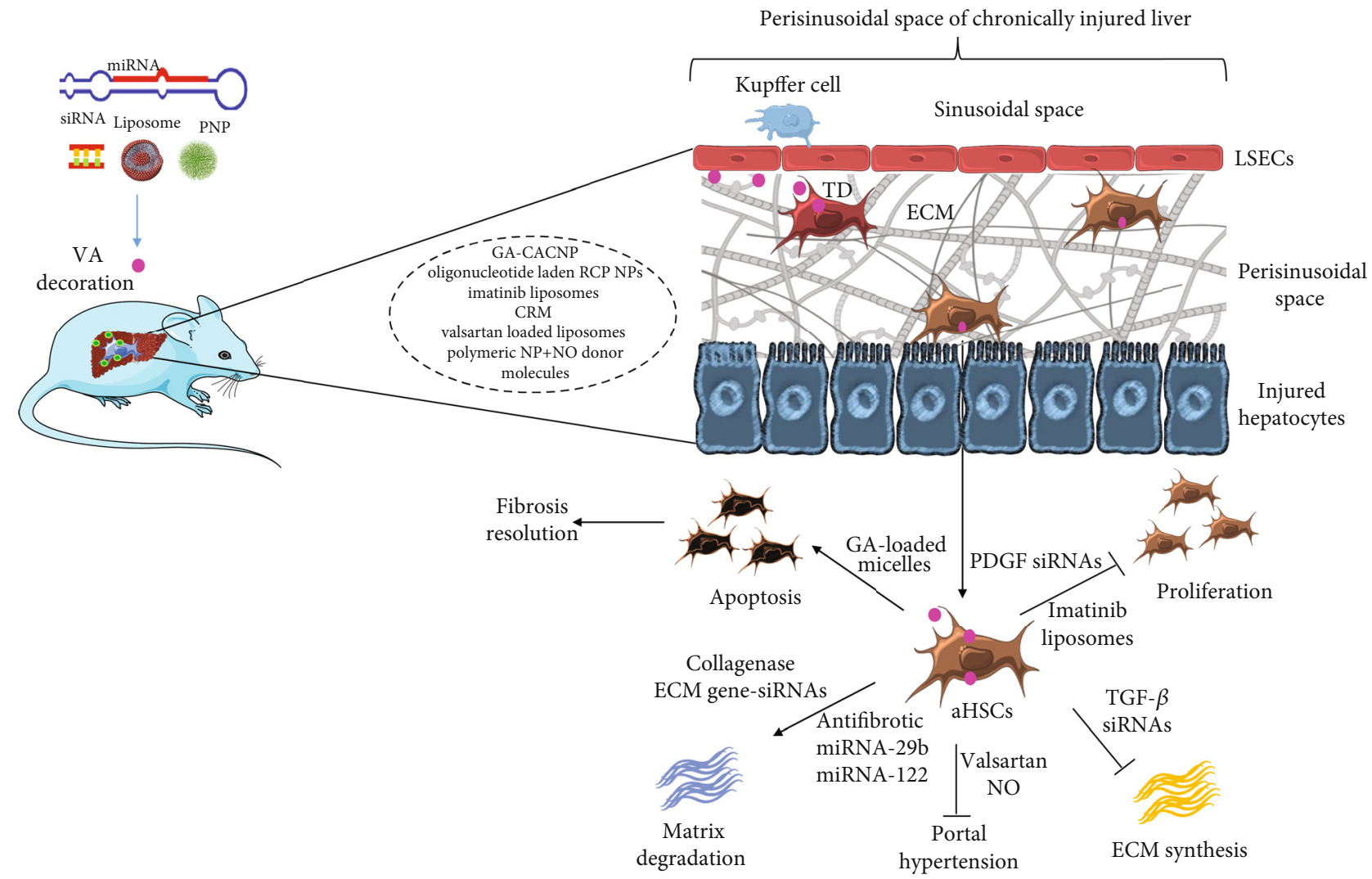

FIGURE 2: Targeted drug delivery for activated HSC (HSCs) in the fibrotic liver. miRNA: microRNA; siRNA: RNA silencing; PNP: polymeric nanoparticles (NPs); VA: vitamin A; GA-CACNP: gallic acid- (GA-) zein/phospholipid composite NPs coupled with VA; RCP NPs: retinolconjugated polyethyleneimine nanoparticles; CRM: collagenase I and retinol codecorated polymeric micelle based on poly-(lactic-coglycolic)-b-poly(ethylene glycol)-maleimide; NO: nitric oxide; LSECs: liver sinusoidal endothelial cells; TD: targeted delivery; ECM: extracellular matrix; PDGF: platelet-derived growth factors; TGF- $\beta$ : transforming growth factor-beta.

significantly after streptavidin, siRNA, cholesterol, and protamine (SSCP) nano-multifaced treatment in activated HSCs [55]. Later, Shukla et al. [56] demonstrated the intracellular transferring of oligonucleotide siRNA and their effective discharge into the HSC cytosol. After internalization, the siRNA nanocomplex with protamine and streptavidin entrapped in early endosomes undergoes exocytosis, entrapment in lysosomes, and endosomal escape. This caused a substantial quantity of siRNA dissociation from the nanocomplex to utilize gene silencing activity.

The IGF2R is increasingly expressed in activated HSCs. Therefore, biotin-conjugated IGF2R-specific peptide, vitamin $\mathrm{A}$, and cholesterol were used as the targeting ligands for the neutravidin-based siRNA nanocomplex to deliver PCBP2 siRNA to activated HSCs. Interestingly, IGF2Rspecific siRNA-neutravidin-peptide-protamine nanocomplex $(228 \mathrm{~nm})$ showed a maximum targeting effect to human (LX-2) and rat (HSC-T6) HSCs and activated initial rat HSCs as compared to cholesterol $(191 \mathrm{~nm})$ and vitamin A $(167 \mathrm{~nm})$ nanocomplexes. IGF2R-specific siRNA-neutravidin-peptide-protamine nanocomplex exhibited the highest liver uptake than lung and kidney in $\mathrm{CCl}_{4}$-induced fibrotic rats. IGF2R-specific siRNA-neutravidin-peptide-protamine nanocomplex had maximum PCBP2 silencing and prevented the migration of activated HSCs [57]. Though
siRNA-neutravidin-peptide-protamine nanocomplex possesses greater than $228 \mathrm{~nm}$ size due to higher molecular weight of the peptide ligand, still they achieved the maximum liver intake. Vitamin A nanocomplexes have less than $200 \mathrm{~nm}$ size as compared to the cholesterol based nonocoplex. However, for liver directing, the avergage particle size was defined to be less than $200 \mathrm{~nm}$, which highly promotes passive liver targeting [39]. Therefore, a study from Zhao et al. [57] suggests that nanoparticles with more than $200 \mathrm{~nm}$ particle size also possibly target the liver.

The chitosan is a natural, recyclable and hydrophilic polymer; it is commonly used in the development of nanoparticles (NPs) for delivery of drug, particularly for nucleic acid and protein delivery [58]. Chitosan nanoparticles have an innate affinity for ECM proteins such as collagen, suggesting that they could be used to transport drugs to fibrotic livers. A previous study developed TGF- $\beta$-siRNA-loaded chitosan NPs (spherical shaped, zeta potential (ZP) of $35 \pm 1 \mathrm{mV}$, and hydrodynamic diameter (HD) $110 \pm 6 \mathrm{~nm}$ ) to promote efficient delivery into activated HSCs via modification with PDGF- $\beta$ binding peptides. The addition of collagenase increased the internalization of TGF- $\beta$-siRNA-rich chitosan NPs and delivered anti-TGF- $\beta 1$ siRNA into activated HSCs in vitro and in vivo. Collagenase-containing nanoparticles may help to degrade the collagen-rich fibrotic scar in the 
chronically injured liver [59]. Tenascin-C (TnC), an ECM protein secreted by HSCs is minimally detected in normal liver cells; however, it is transiently expressed during tissue damage and performs an important role in tumorigenesis and fibrogenesis [60]. TnC promotes HSC activation, migration, and TGF- $\beta$-mediated type I collagen production in the chronically damaged rat liver [61]. In a study, functionalized mesoporous silica NPs (size $40 \pm 5 \mathrm{~nm}$; HD $157 \pm 15 \mathrm{~nm}$ ) were used for the efficient transport and delivery of siTnC in HSCs. Inhibition of TnC by mesoporous silica NP-loaded siTnC prevents HSC activation-mediated cell migration and prevents the elevation of proinflammatory gene expression in macrophages [62].

A wide variety of microRNA (miRNAs) is modulated during HSC activation [8]. Among these, miRNA-122 was identified as the human liver-specific miRNA and miRNA$29 \mathrm{~b}$ was also considered a vital therapeutic focus for liver fibrosis [5]. miRNA-29b and miRNA-122 inhibit HSCs activation and proliferation and collagen synthesis via targeting profibrotic signaling pathways such as hedgehog or TGF$\beta 1 /$ Smad3 pathways [63-65]. Therefore, to selectively target and to deliver these microRNAs into activated HSCs, a pHsensitive, magnetic resonance imaging visible VA decorated with a copolymer polyethylene glycol (PEG)-polyethyleneimine-poly ( $\mathrm{N}-\left(\mathrm{N}^{\prime}, \mathrm{N}^{\prime}\right.$-diisopropylamino ethyl)-co-benzylamine) aspartamide (T-PBP) nanoparticle was developed. It was assembled into MRI contrast agent superparamagnetic iron oxide-patterned cationic micelle for miRNA-29b and miRNA-122 delivery. Due toVA decoration, T-PBP micelle efficiently delivered both miRNAs into HSCs, resulted in synergistic antifibrotic effects through downregulation of HSC activation and ECM-related marker gene expressions ( $\alpha$-SMA and type 1 collagen and TIMP 1 , respectively) [66].

Herbal-based hepatoprotective preparations have poor solubility, bioavailability, and rapid clearance. Therefore, to increase their solubility and bioavailability, nano-based approach is commonly used in experimental studies and tested against fibrosis models [9]. The herbal-based antifibrotic drug nanopreparation may be useful to target HSCs. For instance, a previous study desinged and optimized a reverse micelle-loaded lipid nanocapsule using BoxBehnken design to deliver GA, which has antifibrotic effect, directly into activated HSCs. These $30.35 \pm 2.34 \mathrm{~nm}$-sized GA nanoparticles had high EE $(63.95 \pm 2.98 \% w / w)$ with effective internalization into activated HSCs and reduced their proliferation and induced apoptosis. In vivo, these NPs accumulated in the liver and downregulated the fibrotic marker gene expressions [67]. San-Huang-Xie-Xin-Tang decoction containing Rhei rhizome, Coptidis rhizome, and Scutellariae radix, a traditional herbal medicine of China, is extensively used as hepatoprotective treatment. These dried herbal mixtures were nanoscaled using a pulsed air flow pulverizer. These pulverized nanoscale herbal mixtures induced cytotoxicity in HSCs. This nanoherbal extract has also upregulated MMP-2 gene expression and inhibited TIMP-1 gene expression [68]. However, RNA interference using siRNA and miRNAs encounters many challenges after its systemic administration. Successful and consistent siRNA delivery to activated HSCs in the fibrotic liver remains a vital issue for the synthesis of siRNA-based therapies. The targeted drug delivery for activated HSC in the fibrotic liver is presented in Figure 2.

\section{Conclusion and Perspectives}

Undoubtedly, vitamin A-conjugated nanoparticles or siRNAs or miRNAs or drugs successfully deliver antifibrotic drugs into activated HSCs in the chronically injured liver and reduce profibrotic protein and gene expressions. Vitamin A-conjugated nitric oxide donor molecules and other antihypertensive drugs selectively deliver drugs into activated HSCs and reduce portal hypertension. To achieve better liver targeting, the average particle size was defined to be less than $200 \mathrm{~nm}$, which greatly promotes passive liver targeting. However, Zhao et al. [57] developed a siRNA-neutravidin-peptide-protamine nanocomplex that achieved maximum targeted liver delivery with the particle size of $228 \mathrm{~nm}$. Therefore, size is not the only factor to achieve the highest theranostic potential of a nanocomplex. Hepatic fibrosis is a consequence of the accumulation of numerous ECM proteins in the damaged liver. Therefore, targeting one protein expression via gene silencing using siRNAbased nanoconstruct may not give us efficient results and this is one of the main limitations of siRNA-based therapeutics. However, nanodecoration of HSC-specific molecules provides better siRNA delivery. Further, exocytosis is one of the main factors responsible for the transient silencing activity of non-viral siRNA delivery, and therefore, exocytosis inhibitor combination may prolong the silencing activity of siRNA. However, this approach should be studied in detail in the near future. Moreover, a variety of miRNAs are modulated during HSC activation. They have specific profibrotic and antifibrotic properties; therefore, their specific antagomir- and agomir-based nanopreparations should be studied in an eloborate manner, respectively. Thus, liveror HSC-specific nanopreparation remains a gold standard strategy to deliver siRNA/miRNA/drugs into activated HSCs in the fibrotic liver.

\section{Data Availability}

The data used to support the findings of this study are included within the article

\section{Conflicts of Interest}

The authors declare that they have no conflicts of interest.

\section{References}

[1] S. K. Asrani, H. Devarbhavi, J. Eaton, and P. S. Kamath, "Burden of liver diseases in the world," Journal of Hepatology, vol. 70, no. 1, pp. 151-171, 2019.

[2] A. K. Singal and P. Mathurin, "Diagnosis and treatment of alcohol-associated liver disease: a review," Journal of the American Medical Association, vol. 326, no. 2, pp. 165-176, 2021.

[3] E. Devaraj and S. Rajeshkumar, "Nanomedicine for hepatic fibrosis," in Nanoparticles and their Biomedical Applications, A. Shukla, Ed., Springer, Singapore, 2020. 
[4] D. Ezhilarasan, "Critical role of estrogen in the progression of chronic liver diseases," Hepatobiliary \& Pancreatic Diseases International, vol. 19, no. 5, pp. 429-434, 2020.

[5] D. Ezhilarasan, "MicroRNA interplay between hepatic stellate cell quiescence and activation," European Journal of Pharmacology, vol. 885, article 173507, 2020.

[6] A. Khanam, P. G. Saleeb, and S. Kottilil, "Pathophysiology and treatment options for hepatic fibrosis: can it be completely cured?," Cell, vol. 10, no. 5, p. 1097, 2021.

[7] D. Schuppan, M. Ashfaq-Khan, A. T. Yang, and Y. O. Kim, "Liver fibrosis: direct antifibrotic agents and targeted therapies," Matrix Biology, vol. 68-69, pp. 435-451, 2018.

[8] D. Ezhilarasan, "Oxidative stress is bane in chronic liver diseases: clinical and experimental perspective," Arab Journal of Gastroenterology, vol. 19, no. 2, pp. 56-64, 2018.

[9] D. Ezhilarasan, E. Sokal, S. Karthikeyan, and M. Najimi, "Plant derived antioxidants and antifibrotic drugs: past, present and future," Journal of Coastal Life Medicine, vol. 2, no. 9, pp. 738-745, 2014.

[10] T. Tsuchida and S. L. Friedman, "Mechanisms of hepatic stellate cell activation," Nature Reviews. Gastroenterology \& Hepatology, vol. 14, no. 7, pp. 397-411, 2017.

[11] P. Königshofer, K. Brusilovskaya, O. Petrenko et al., "Nuclear receptors in liver fibrosis," Biochimica et Biophysica Acta Molecular Basis of Disease, vol. 1867, no. 12, article 166235, 2021.

[12] C. Ortiz, R. Schierwagen, L. Schaefer, S. Klein, X. Trepat, and J. Trebicka, "Extracellular matrix remodeling in chronic liver disease," Current Tissue Microenvironment Reports, vol. 2, no. 3, pp. 41-52, 2021.

[13] S. Kumar, Q. Duan, R. Wu, E. N. Harris, and Q. Su, "Pathophysiological communication between hepatocytes and non-parenchymal cells in liver injury from NAFLD to liver fibrosis," Advanced Drug Delivery Reviews, vol. 176, article 113869, 2021.

[14] X. Cai, J. Wang, J. Wang et al., "Intercellular crosstalk of hepatic stellate cells in liver fibrosis: new insights into therapy," Pharmacological Research, vol. 155, p. 104720, 2020.

[15] D. Ezhilarasan, "Endothelin-1 in portal hypertension: the intricate role of hepatic stellate cells," Experimental Biology and Medicine (Maywood, N.J.), vol. 245, no. 16, pp. 15041512, 2020.

[16] D. Ezhilarasan, "Role of microRNAs in hepatic fibrosis progression," Journal of Applied Pharmaceutical Science, vol. 8, no. 5, pp. 174-178, 2018.

[17] E. Borkham-Kamphorst, J. Herrmann, D. Stoll, J. Treptau, A. M. Gressner, and R. Weiskirchen, "Dominant-negative soluble PDGF- $\beta$ receptor inhibits hepatic stellate cell activation and attenuates liver fibrosis," Laboratory Investigation, vol. 84, no. 6, pp. 766-777, 2004.

[18] A. Baiocchini, C. Montaldo, A. Conigliaro et al., "Extracellular matrix molecular remodeling in human liver fibrosis evolution," PLoS One, vol. 11, no. 3, article e0151736, 2016.

[19] D. Ezhilarasan, J. Evraerts, S. Brice et al., "Silibinin inhibits proliferation and migration of human hepatic stellate LX-2 cells," Journal of Clinical and Experimental Hepatology, vol. 6, no. 3, pp. 167-174, 2016.

[20] H. Nishizawa, G. Iguchi, H. Fukuoka et al., "IGF-I induces senescence of hepatic stellate cells and limits fibrosis in a p53-dependent manner," Scientific Reports, vol. 6, no. 1, p. ???, 2016.
[21] R. Brea, O. Motiño, D. Francés et al., " $\mathrm{PGE}_{2}$ induces apoptosis of hepatic stellate cells and attenuates liver fibrosis in mice by downregulating miR-23a-5p and miR-28a-5p," Biochimica et Biophysica Acta - Molecular Basis of Disease, vol. 1864, no. 2, pp. 325-337, 2018.

[22] P. Gupta, T. N. Sata, A. K. Yadav et al., “TGF- $\beta$ induces liver fibrosis via miRNA-181a-mediated down regulation of augmenter of liver regeneration in hepatic stellate cells," PLoS One, vol. 14, no. 6, article e0214534, 2019.

[23] D. Ezhilarasan, J. Evraerts, B. Sid et al., "Silibinin induces hepatic stellate cell cycle arrest via enhancing p53/p27 and inhibiting Akt downstream signaling protein expression," Hepatobiliary \& Pancreatic Diseases International, vol. 16, no. 1, pp. 80-87, 2017.

[24] D. Ezhilarasan, "Hepatic stellate cells in the injured liver: perspectives beyond hepatic fibrosis," Journal of Cellular Physiology, 2021.

[25] H. Zhang, W. Han, X. Cao et al., "Gold nanoclusters as a nearinfrared fluorometric nanothermometer for living cells," Mikrochimica Acta, vol. 186, no. 6, p. 353, 2019.

[26] F. Peng, J. K. Tee, M. I. Setyawati et al., "Inorganic nanomaterials as highly efficient inhibitors of cellular hepatic fibrosis," ACS Applied Materials \& Interfaces, vol. 10, no. 38, pp. 31938-31946, 2018.

[27] D. Yang, Y. H. Gao, K. B. Tan et al., "Inhibition of hepatic fibrosis with artificial microRNA using ultrasound and cationic liposome-bearing microbubbles," Gene Therapy, vol. 20, no. 12, pp. 1140-1148, 2013.

[28] S. Poilil Surendran, R. George Thomas, M. J. Moon, and Y. Y. Jeong, "Nanoparticles for the treatment of liver fibrosis," International Journal of Nanomedicine, vol. 12, pp. 6997-7006, 2017.

[29] Z. Chen, A. Jain, H. Liu, Z. Zhao, and K. Cheng, “Targeted drug delivery to hepatic stellate cells for the treatment of liver fibrosis," The Journal of Pharmacology and Experimental Therapeutics, vol. 370, no. 3, pp. 695-702, 2019.

[30] X. Zhou, F. R. Murphy, N. Gehdu, J. Zhang, J. P. Iredale, and R. C. Benyon, "Engagement of $\alpha_{v} \beta_{3}$ Integrin Regulates Proliferation and Apoptosis of Hepatic Stellate Cells*," The Journal of Biological Chemistry, vol. 279, no. 23, pp. 23996-24006, 2004.

[31] T. Li, X. S. Leng, J. Y. Zhu, and G. Wang, "Suppression of hedgehog signaling regulates hepatic stellate cell activation and collagen secretion," International Journal of Clinical and Experimental Pathology, vol. 8, no. 11, pp. 14574-14579, 2015.

[32] Y. Li, S. Pu, Q. Liu et al., “An integrin-based nanoparticle that targets activated hepatic stellate cells and alleviates liver fibrosis," Journal of Controlled Release, vol. 303, pp. 77-90, 2019.

[33] L. Tao, L. Wu, W. Zhang et al., "Peroxisome proliferatoractivated receptor $\gamma$ inhibits hepatic stellate cell activation regulated by miR-942 in chronic hepatitis B liver fibrosis," Life Sciences, vol. 253, p. 117572, 2020.

[34] S. C. Zhi, S. Z. Chen, Y. Y. Li, J. J. Li, Y. H. Zheng, and F. X. Yu, "Rosiglitazone inhibits activation of hepatic stellate cells via up-regulating micro-RNA-124-3p to alleviate hepatic fibrosis," Digestive Diseases and Sciences, vol. 64, no. 6, pp. 1560-1570, 2019.

[35] V. Kumar, V. Mundra, and R. I. Mahato, "Nanomedicines of hedgehog inhibitor and PPAR- $\gamma$ agonist for treating liver fibrosis," Pharmaceutical Research, vol. 31, no. 5, pp. 1158 1169, 2014. 
[36] Q. Zhang, D. Xu, Q. Guo et al., "Theranostic quercetin nanoparticle for treatment of hepatic fibrosis," Bioconjugate Chemistry, vol. 30, no. 11, pp. 2939-2946, 2019.

[37] D. Ji, Q. Wang, Q. Zhao et al., "Co-delivery of miR-29b and germacrone based on cyclic RGD-modified nanoparticles for liver fibrosis therapy," Journal of Nanobiotechnology, vol. 18, no. 1, p. $86,2020$.

[38] M. W. Haaker, A. B. Vaandrager, and J. B. Helms, "Retinoids in health and disease: a role for hepatic stellate cells in affecting retinoid levels," Biochimica et Biophysica Acta-Molecular and Cell Biology of Lipids, vol. 1865, no. 6, article 158674, 2020.

[39] D. Ezhilarasan, "Advantages and challenges in nanomedicines for chronic liver diseases: A hepatologist's perspectives," European Journal of Pharmacology, vol. 893, article 173832, 2021.

[40] S. A. A. Radwan, W. H. El-Maadawy, A. N. ElMeshad, R. A. Shoukri, and C. Yousry, "Impact of reverse micelle loaded lipid nanocapsules on the delivery of gallic acid into activated hepatic stellate cells: a promising therapeutic approach for hepatic fibrosis," Pharmaceutical Research, vol. 37, no. 9, p. 180, 2020.

[41] Z. Zhang, C. Wang, Y. Zha et al., "Corona-directed nucleic acid delivery into hepatic stellate cells for liver fibrosis therapy," ACS Nano, vol. 9, no. 3, pp. 2405-2419, 2015.

[42] M. C. Xiao, H. Qian, C. K. Huang et al., "Imatinib inhibits the malignancy of hepatocellular carcinoma by suppressing autophagy," European Journal of Pharmacology, vol. 906, article 174217, 2021.

[43] K. Qu, Z. Huang, T. Lin et al., "New insight into the anti-liver fibrosis effect of multitargeted tyrosine kinase inhibitors: from molecular target to clinical trials," Frontiers in Pharmacology, vol. 6, p. 300, 2016.

[44] N. S. El-Mezayen, W. F. El-Hadidy, W. M. El-Refaie, T. I. Shalaby, M. M. Khattab, and A. S. El-Khatib, "Hepatic stellate celltargeted imatinib nanomedicine versus conventional imatinib: A novel strategy with potent efficacy in experimental liver fibrosis," Journal of Controlled Release, vol. 266, pp. 226-237, 2017.

[45] Q. Q. Fan, C. L. Zhang, J. B. Qiao et al., "Extracellular matrixpenetrating nanodrill micelles for liver fibrosis therapy," Biomaterials, vol. 230, p. 119616, 2020.

[46] M. Yalniz, A. Demir, A. Arslan, and M. Cihangiroğlu, "Short term effects of valsartan on portal blood flow in cirrhotic patients," The Turkish Journal of Gastroenterology, vol. 14, no. 1, pp. 18-25, 2003.

[47] C. Fierbinteanu-Braticevici, P. Dragomir, L. Tribus et al., "The effect of valsartan, an angiotensin II receptor antagonist, on portal and systemic hemodynamics and on renal function in liver cirrhosis," Journal of Gastrointestinal and Liver Diseases, vol. 15, no. 4, pp. 337-342, 2006.

[48] L. J. Huo, Y. Liu, and S. J. Zhang, "Effect of valsartan on portal pressure and hepatic fibrosis in patients with hepatic cirrhosis," World Chinese Journal of Digestology, vol. 17, no. 11, pp. 1139-1142, 2009.

[49] G. Flesch, P. Müller, and P. Lloyd, “Absolute bioavailability and pharmacokinetics of valsartan, an angiotensin II receptor antagonist, in man," European Journal of Clinical Pharmacology, vol. 52, no. 2, pp. 115-120, 1997.

[50] N. S. El-Mezayen, W. F. El-Hadidy, W. M. El-Refaie, T. I. Shalaby, M. M. Khattab, and A. S. El-Khatib, "Oral vitamin-A-coupled valsartan nanomedicine: high hepatic stellate cell receptors accessibility and prolonged enterohepatic residence," Journal of Controlled Release, vol. 283, pp. 32-44, 2018.
[51] A. Das, P. Mukherjee, S. K. Singla et al., "Fabrication and characterization of an inorganic gold and silica nanoparticle mediated drug delivery system for nitric oxide," Nanotechnology, vol. 21, no. 30, p. 305102, 2010.

[52] H. T. Duong, Z. Dong, L. Su et al., "The use of nanoparticles to deliver nitric oxide to hepatic stellate cells for treating liver fibrosis and portal hypertension," Small, vol. 11, no. 19, pp. 2291-2304, 2015.

[53] R. Omar, J. Yang, H. Liu, N. M. Davies, and Y. Gong, "Hepatic stellate cells in liver fibrosis and siRNA-based therapy," Reviews of Physiology, Biochemistry and Pharmacology, vol. 172, pp. 1-37, 2016.

[54] Z. Zhao, C. Y. Lin, and K. Cheng, "siRNA- and miRNA-based therapeutics for liver fibrosis," Translational Research, vol. 214, pp. 17-29, 2019.

[55] R. S. Shukla, B. Qin, Y. J. Wan, and K. Cheng, "PCBP2 siRNA reverses the alcohol-induced pro-fibrogenic effects in hepatic stellate cells," Pharmaceutical Research, vol. 28, no. 12, pp. 3058-3068, 2011.

[56] R. S. Shukla, A. Jain, Z. Zhao, and K. Cheng, "Intracellular trafficking and exocytosis of a multi-component siRNA nanocomplex," Nanomedicine, vol. 12, no. 5, pp. 1323-1334, 2016.

[57] Z. Zhao, Y. Li, A. Jain et al., "Development of a peptidemodified siRNA nanocomplex for hepatic stellate cells," Nanomedicine, vol. 14, no. 1, pp. 51-61, 2018.

[58] R. C. Cheung, T. B. Ng, J. H. Wong, and W. Y. Chan, "Chitosan: an update on potential biomedical and pharmaceutical applications," Marine Drugs, vol. 13, no. 8, pp. 5156-5186, 2015.

[59] M. Azzam, S. El Safy, S. A. Abdelgelil et al., "Targeting activated hepatic stellate cells using collagen-binding chitosan nanoparticles for siRNA delivery to fibrotic livers," Pharmaceutics, vol. 12, no. 6, p. 590, 2020.

[60] A. El-Karef, M. Kaito, H. Tanaka et al., "Expression of large tenascin-C splice variants by hepatic stellate cells/myofibroblasts in chronic hepatitis C," Journal of Hepatology, vol. 46, no. 4, pp. 664-673, 2007.

[61] J. C. Ma, X. Huang, Y. W. Shen et al., "Tenascin-C promotes migration of hepatic stellate cells and production of type I collagen," Bioscience, Biotechnology, and Biochemistry, vol. 80, no. 8, pp. 1470-1477, 2016.

[62] J. L. Vivero-Escoto, H. Vadarevu, R. Juneja, L. W. Schrum, and J. H. Benbow, "Nanoparticle mediated silencing of tenascin C in hepatic stellate cells: effect on inflammatory gene expression and cell migration," Journal of Materials Chemistry B, vol. 7, no. 46, pp. 7396-7405, 2019.

[63] J. Li, M. Ghazwani, Y. Zhang et al., "miR-122 regulates collagen production via targeting hepatic stellate cells and suppressing P4HA1 expression," Journal of Hepatology, vol. 58, no. 3, pp. 522-528, 2013.

[64] J. Wang, E. S. Chu, H. Y. Chen et al., "microRNA-29b prevents liver fibrosis by attenuating hepatic stellate cell activation and inducing apoptosis through targeting PI3K/AKT pathway," Oncotarget, vol. 6, no. 9, pp. 7325-7338, 2015.

[65] C. Liang, S. Bu, and X. Fan, "Suppressive effect of microRNA$29 \mathrm{~b}$ on hepatic stellate cell activation and its crosstalk with TGF- $\beta 1 /$ Smad3," Cell Biochemistry and Function, vol. 34, no. 5 , pp. 326-333, 2016.

[66] J. Wu, J. Huang, S. Kuang et al., "Synergistic microRNA therapy in liver fibrotic rat using MRI-visible nanocarrier targeting hepatic stellate cells," Advanced Science, vol. 6, no. 5, article 1801809, 2019. 
[67] S. A. A. Radwan, W. H. El-Maadawy, C. Yousry, A. N. ElMeshad, and R. A. Shoukri, "Zein/Phospholipid composite nanoparticles for successful delivery of gallic acid into aHSCs: influence of size, surface charge, and vitamin a Coupling," International Journal of Nanomedicine, vol. 15, pp. 7995-8018, 2020.

[68] S. Huang, S. J. Chang, M. Yang, J. J. Chen, and W. H. Chang, "Nanoscale hepatoprotective herbal decoction attenuates hepatic stellate cell activity and chloroform-induced liver damage in mice," International Journal of Nanomedicine, vol. 6, pp. 1365-1371, 2011. 